\title{
Structural analysis of the phimotic prepuce in patients with failed topical treatment compared with untreated phimosis
}

Luciano Alves Favorito, Carlos M. Balassiano, João Pedro Rosado, Luiz Eduardo M. Cardoso, Waldemar Silva Costa, Francisco José Barcellos Sampaio

Urogenital Research Unit, State University from Rio de Janeiro, Brazil

ABSTRACT

Objectives: To evaluate histological alterations in prepuce of patients with phimosis submitted to topic treatment with betamethasone in association with hyaluronidase. Materials and Methods: We studied sixty patients (mean age 4.5), presenting true phimosis and treated with a topical treatment with betamethasone cream $(0.2 \%)+$ hyaluronidase. The parents of seven of these patients opted for circumcision (control group). The other fifty-three patients were submitted to clinical treatment. The samples were stained with Weigert's resorcin-fuchsin (analysis of the elastic fibers) and Picro-Sirius Red, for analysis of the collagen. The volumetric density of the elastic fibers was determined by stereological methods.

Results: Only eight (15\%) of the fifty-three patients submitted to topical treatment presented failure, being indicated for circumcision (histological analysis). We observed an increase of the collagen type III of the patients submitted to topical treatment. The quantification showed a reduction of the volumetric density of the prepuce's elastic fibers of the patients submitted to the cream treatment, when compared to the control group ( $p=0.056)$. The volumetric density of the elastic fibers of the prepuce at the group not submitted to topical treatment showed an average of 14.60\% (11.06 to $21.64 \%$ ); in the group submitted to the cream treatment, the volumetric density of the elastic fibers of the prepuce showed an average of 10.34\% (3.45 to 17.9\%).

Conclusion: The topical treatment of phimosis with betamethasone $0.2 \%+$ hyaluronidase had a success rate of $85 \%$. Patients with failure of the topical treatment with steroid had histological alterations in the prepuce.

\section{ARTICLE INFO}

\section{Key words:}

Phimosis; Methods; Anatomy

and histology; Histology;

Circumcision

Int Braz J Urol. 2012; 38: 802-8

Submitted for publication:

January 19, 2012

Accepted after revision:

May 22, 2012

\section{INTRODUCTION}

The foreskin is a specialized and innervated mucous-cutaneous tissue that covers and protects the glans. After 3 years of age, cysts of keratin are formed below the foreskin adherences and together with intermittent erections are able to enlarge the phimotic ring, exposing the glans. Around 80 to $90 \%$ of the boys that were not circu- mcised became capable to expose the glans after 3 years of age (1).

Pathological phimosis is characterized by a foreskin fibrotic ring with adherences that does not allow the exposition of the glans (1). This alteration hinders adequate penile hygiene that favors foreskin infections, repeated urinary infections, sexually transmitted diseases and, at adult age, penile carcinoma (2). 
Circumcision at childhood must be performed under general anesthesia. Moreover, the procedure itself is not free of risks, presenting complication rates up to $34 \%(3,4)$. The most common complications of circumcision are hemorrhage, urethral meatus and preputial ring stenosis, and even glans amputation (5). Besides these problems, circumcision presents considerable costs, which could approach a mean of US\$ $1,920.00$ per procedure, according to recent reports $(6,7)$.

The clinical treatment of phimosis with topical corticosteroids has been proposed as an alternative to surgery in the beginning of 1990, demonstrating acceptable results (8-10) and low cost (11). Since then, several authors have presented results varying from $67 \%$ to $95 \%$ of sucess, independently of the patients' age. The most common used topical treatments were betamethasone, clobetasol, diclofenac sodium, mometasone furoate $0.05 \%$ and triamcinolone acetate $(10,12,13)$. Recent randomized prospective studies have confirmed that topical use of corticosteroids as treatment for phimosis is superior to placebo $(14,15)$.

Studies on the foreskin structure in patients with phimosis are scarce. Also, to our knowledge, there are no studies on histological alterations of the foreskin after topical corticosteroids treatment. Therefore, the objective of the present work was to evaluate the possible histological alterations in the foreskin of patients with phimosis submitted to topical application of an association of betamethasone $0.2 \%$ and hyaluronidase cream.

\section{MATERIALS AND METHODS}

The present work received institutional committee review and parental approval. This work was carried out in accordance with the ethical standards of the institutional committee responsible for human experimentation.

We studied 60 patients ranging from 3 to 10 years (mean 4.5), during the period from January 2006 to October 2010. All patients presented true phimosis with foreskin stenosis and attended the pediatric urology ambulatory care to be submitted to circumcision. All 60 patients presented total impossibility of foreskin retraction, which characterizes the type A foreskin anatomy accor- ding to Marques (16). The exclusion criteria were patients with less than 3 years of age, patients without true phimosis and patients with clinical suspicion of balanitis xerotica obliterans.

To the parents of the boys referred for circumcision we offered an alternative clinical management of the phimosis. The regimen proposed was a topical application of an association of betamethasone $0.2 \%$ and hyaluronidase (150 units of turbid retention) cream on the phimotic ring. The parents were oriented to perform gentle traction of the foreskin until the stenotic ring could be seen. The cream was applied twice a day, for 6 consecutive weeks, associated with appropriate hygiene of the penis. The children had a follow up every 3 weeks in our ambulatory.

The parents of 7 children did not agree with the clinical treatment and opted for circumcision directly. These 7 patients served as the control group and the final treated sample was composed of 53 patients.

The topical treatment was considered successful if the patient was able to expose the glans completely. We considered failure if the glans could not be exposed after treatment or if occurred foreskin infection during treatment. In these cases, circumcision was performed. The patients submitted to topical treatment were followed up for 6 months.

\section{Histological Analysis}

In controls and in those cases on which the patients failed treatment and were submitted to circumcision, the foreskin was fixed in a 10\% buffered formalin solution, routinely processed for embedding in paraffin and 5- $\mu \mathrm{m}$ thick sections were obtained. The sections were stained with Picro-Sirius Red, for qualitative analysis of the collagen under microscope of polarization and Weigert's Resorcin Fuscin with previous oxidation by oxona, for characterization and quantification of the elastic system fibers.

\section{Quantitative Analysis}

From each specimen (foreskin), 5 different sections were randomly selected. From each section, 5 random fields were analyzed, totaling 25 fields (test areas) for each specimen. The data on 
elastic system fibers were expressed as volumetric density ( $\mathrm{Vv}-\%)$. The sections were observed with X400 magnification using an Olympus light microscope coupled to a video camera Sony CCD, and the images transferred to a Sony monitor KX14-CP1. The selected histological areas were then quantified using M42 test-grid system on the digitized fields $(17,18)$. (Figure-1). All numerical results are presented as mean \pm standard deviation.

The data were analyzed with the Graphpad software. To compare the quantitative data in both groups (controls and treated) and the outcomes, the Student's t-test was used ( $p<0.05$ was considered significant) (19).

\section{RESULTS}

From the 53 patients submitted to topical treatment with the association of betamethasone and hyaluronidase cream, $8(15 \%)$ presented failure $(6$ could not expose the glans and 2 remained with foreskin stenosis) and were referred to circumcision. The foreskin of these patients was submitted to histological analysis and composed our treated group. The others 45 patients presented significant improvement of the phimosis, with total exposure of the glans without foreskin stenosis. After 6 months of follow-up, these patients considered as success did not present recurrence of the phimosis.

We analyzed the alterations on the elastic fibers and collagen of the foreskin. Elastic system fibers were analyzed in the foreskin of the control $(\mathrm{n}=7)$ and treatment failed $(\mathrm{n}=8)$ groups. Figure-2 shows the elastic system fibers analysis in the foreskin. One may note in Figure-2B, in a patient who failed the topical treatment, an apparent lower amount of elastic fibers when compared to controls (Figure-2A). The quantification demonstrated that the mean volumetric density of elastic fibers in the control group was $14.60 \%$ (11.06 to $21.64 \%$ ) and in the group who failed treatment was $10.34 \%$ (3.45 to $17.9 \%$ ); nevertheless, the difference was not significant $(p=0.056)$. Data on the stereological quantification of elastic system fibers in the foreskin are presented in details on Table- 1 .

The Figure-3 evidences the alterations on the concentration of the collagen of the two groups studied, through the PicroSirius Red stain (with polarization). We can see the alteration on the pattern of the collagen of the patients submitted to the treatment with the cream. In Figure-3B,

Figure 1 - Quantification of elastic fibers: The quantification was obtained using the M42 Test system. Photomicrograph of the prepuce of an 8-year old patient of the control group (without treatment). Weigert stain reduced from X400.

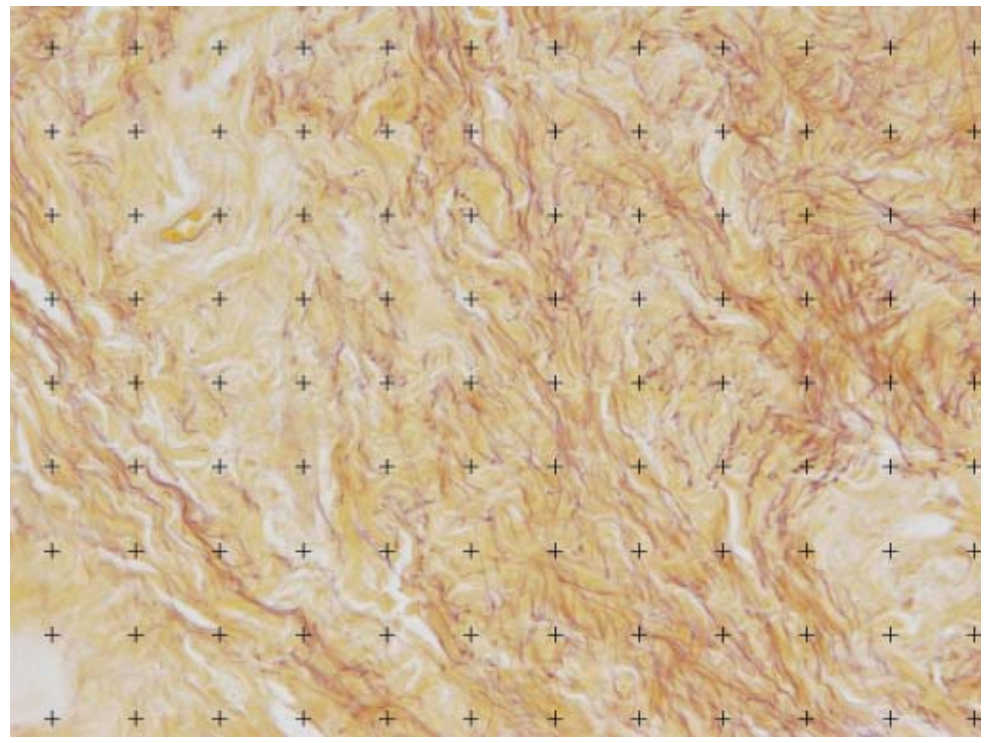


Figure 2 - Analysis of the elastic fibers: A) Photomicrography of the prepuce of a 3-year old patient not treated with topical steroid. It is possible to observe that the elastic fibers (purple) are distributed over the whole field. Weigert stain reduced X400. B) Photomicrography of the prepuce of a 3-year old patient submitted to topical steroid treatment. It is possible to oberserve the elastic fibers (purple). Weigert stain reduced X400.

A

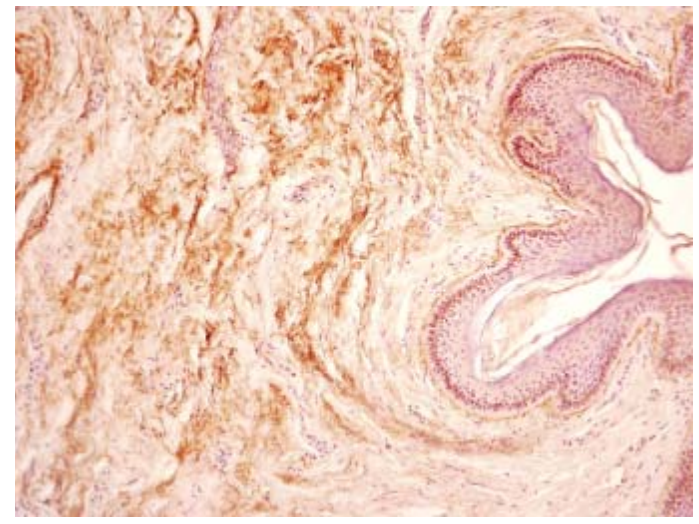

B

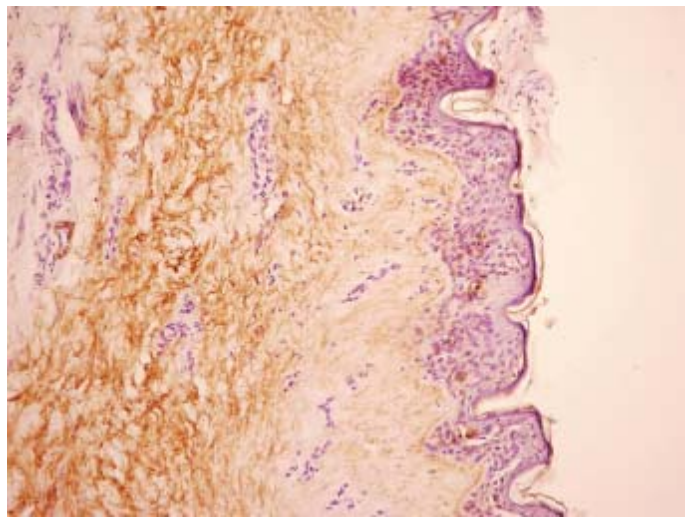

Table 1 - Quantification of elastic system fibers in the foreskin of controls $(n=7)$ and of patients who failed topical treatment $(\mathrm{n}=8)$ with the association of betamethasone and hyaluronidase cream.

\begin{tabular}{|c|c|c|c|c|}
\hline Elastic System Fibers & $\begin{array}{c}\text { Mean }(\%) \pm \text { Standard } \\
\text { Deviation }\end{array}$ & Maximum & Minimum & Variance \\
\hline Controls & $14.61 \pm 4.106$ & 21.64 & 11.06 & 1.552 \\
\hline Failed Treatment & $10.35 \pm 5.247$ & 17.90 & 3.45 & 1.855 \\
\hline
\end{tabular}

$p=0.056$; not significant.

Figure 3 - Qualitative analysis of the collagen: A) Photomicrography of the prepuce of a 4 year old patient without treatment with topical steroid. Collagen type I - red; Collagen type III - green. Picro-Sirius Red stain reduced X 400. B) Photomicrography of the prepuce of a 4 year old patient of the group submitted to topical steroid treatment. It is possible to observe an enlargement of green color (collagen type III), in this group. Picro-Sirius Red stain reduced X 400.

A

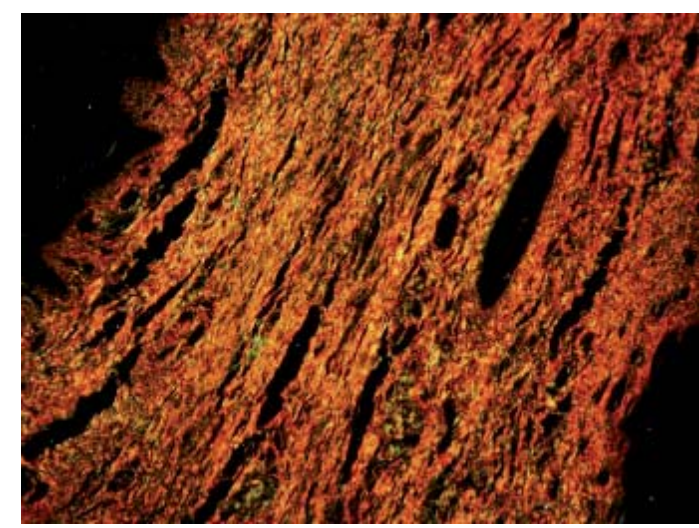

B

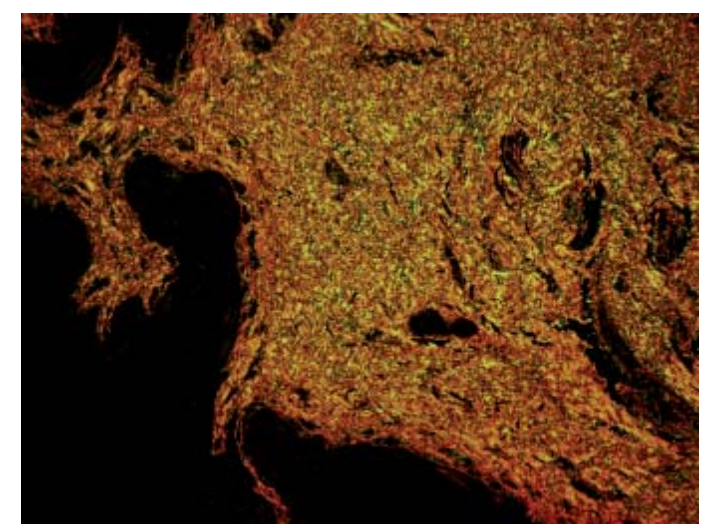


one may observe the increase of the collagen type III (colored in green), on patients submitted to treatment with topical steroid.

\section{DISCUSSION}

The physiological phimosis affects $96 \%$ of the newborns and its incidence reduces with age. At 3 years of age, 10\% of the patients present phimosis and at 14 years of age, this incidence affects only $1 \%$ (20). The natural process of enlargement of the prepuce can suffer alterations when facing episodes of balanoposthitis and lesion of the prepuce by traction of the ring that leads to the formation of a healing fibrosis and the impossibility to expose the glans.

The clinical treatment of the phimosis with topic corticosteroids is well accepted by the parents, since it is a simple procedure, presents low costs and risks, no side effects and a good compliance to the treatment when the guardians of the patients are well oriented $(14,15)$. In our studies we did not have reports of significant side effects and all fifty-three patients submitted to clinical treatment completed the six weeks of treatment.

The success rate of the phimosis topic treatment with corticosteroids is significant, with satisfactory results (67\%-95\%) (8-10,14,15). The success rate of the topical treatment of the phimosis is lower when smaller concentrations of betamethasone are used (21). We opted for the use of a larger concentration of betamethasone (0.2\%) associated with hyluronidase. This larger concentration of corticosteroids does not present a larger rate of side effects and could enhance the success rate of the treatment. After six weeks of treatment, among the fifty-three patients treated, 85\% showed significant improvement and were not submitted to surgery, demonstrating the efficiency of the treatment.

Betamethasone is one of the substances that present better rates of efficiency as a topical treatment of phimosis $(20,22)$, therefore the chosen medication in this study. Corticosteroids reduce the arachidonic and hydroxyecosatetranoic acids on the reproductive inflammatory skin disease, inhibiting the liberation of prostaglandins and enlarging the level of dismutase super-oxida- tive activity, being able to liberate anti-oxidants (20). Side effects, such as the suppression of the hypothalamo-pituitary-adrenal axis or cutaneous atrophy, may ocurr; nevertheless, the doses used in the topical treatment of the phimosis are not sufficient to lead to this type of complications (1).

Hyaluronidase is an enzyme obtained from cattle testicles, which depolymerize hyaluronic acid, a mucopolysaccharide that is present in the interstitial tissue spaces (conjunctive tissue of the preputial adherences). The depolimerization of the hyaluronic acid reduces the inter-cellular viscosity, allowing the tissue to remain available to the dispersion of substances. Besides that, hyaluronidase presents elastic cohesion functions, being applied with the objective of cleaning the adherences and also because it presents the effects of a local anesthesia.

Collagen and elastic fibers are the fibrotic components of the cellular matrix and are related to pathological alterations in different tissues. In our studies, we noticed a reduction of volumetric density of the elastic fibers of the patient's prepuces submitted to topical treatment, with corticosteroids + hyaluronidase.

The reduction of elastic fibers of the prepuce in patients submitted to topical treatment with the cream was not significant but is typical of the healing process of this treatment (23). The increasing concentration of elastic fibers is related to a larger widening of the tissues. For an easy exposure of the glans, the prepuce needs a larger concentration of elastic fibers (24). The reduction of the elastic fibers in the prepuce of the patients presenting phimosis and submitted to a betamethasone + hyaluronidase treatment is similar to what occurs in the healing process and could be related to a larger difficulty of the exposure of the glans; these patients did not report infections of the prepuce.

Topical treatment with steroids would intuitively reduce tissue scarring/fibrosis, and therefore is the mechanism by which the treatment would be successful. The interesting question to ask would be why did this cohort of patients have a significant reduction in elastic fibers but still fail topical treatment? These alterations of the elastic system could be involved on the therapeutic failu- 
re of the cream applied; nevertheless, to confirm this hypothesis, it would be necessary to analyze the prepuces of the patients that received the treatment which obviously, for ethical reasons, is not possible.

The use of the betamethasone + hyaluronidase cream led to an evident alteration in the distribution of the collagen in the prepuces submitted to this topical treatment. There was a reduction of the collagen in the prepuce of the patients that made use of it. Nevertheless, when we analyzed the prepuces with the Picrosirus stain, it could be noticed a clear alteration in the distribution of the collagen, with and increase of collagen type III. The greenish fibers that appear under the Picrosirus stain characterize the collagen type III, a recent collagen which is probably produced by muscular retraction. In the control group there was a predominance of a clear reddish color that characterizes the predominance of the collagen type I. It is very difficult to say, without studying the histology of the patients that made use of the cream and did not need a surgery, if the alterations of the collagen occurred due to induced alterations provoked by the cream or due to tissue alterations of the prepuce resulting from local infections.

\section{CONCLUSIONS}

The topical treatment of phimosis with betamethasone $0.2 \%$ + hyaluronidase was effective with a success rate of 85\%. Patients in whom topical steroid treatment failed had fewer elastic fibers, which characterize the healing processes, as well as an amplification of the collagen type III, a recently found collagen that is associated with muscular retraction. The betamethasone + hyaluronidase cream leads to significant histological alterations in the prepuce.

\section{ACKNOWLEDGEMENTS}

Supported by grants from the National Council of Scientific and Technological Development (CNPQ - Brazil) and Foundation for Research Support of Rio de Janeiro (FAPERJ).

\section{CONFLICT OF INTEREST}

\author{
None declared.
}

\section{REFERENCES}

1. Orsola A, Caffaratti J, Garat JM: Conservative treatment of phimosis in children using a topical steroid. Urology. 2000; 56: 307-10.

2. Elmore JM, Baker LA, Snodgrass WT: Topical steroid therapy as an alternative to circumcision for phimosis in boys younger than 3 years. J Urol. 2002; 168(4 Pt 2): 1746-7; discussion 1747.

3. Chu CC, Chen KC, Diau GY: Topical steroid treatment of phimosis in boys. J Urol. 1999; 162(3 Pt 1): 861-3.

4. Cathcart P, Nuttall M, van der Meulen J, Emberton M, Kenny SE: Trends in paediatric circumcision and its complications in England between 1997 and 2003. Br J Surg. 2006; 93: 885-90.

5. Ozkan S, Gürpinar T: A serious circumcision complication: penile shaft amputation and a new reattachment technique with a successful outcome. J Urol. 1997; 158: 1946-7.

6. Berdeu D, Sauze L, Ha-Vinh P, Blum-Boisgard C: Costeffectiveness analysis of treatments for phimosis: a comparison of surgical and medicinal approaches and their economic effect. BJU Int. 2001; 87: 239-44.

7. Schoen EJ, Colby CJ, To TT: Cost analysis of neonatal circumcision in a large health maintenance organization. $J$ Urol. 2006; 175(3 Pt 1): 1111-5.

8. Golubovic Z, Milanovic D, Vukadinovic V, Rakic I, Perovic $\mathrm{S}$ : The conservative treatment of phimosis in boys. $\mathrm{Br} \mathrm{J}$ Urol. 1996; 78: 786-8.

9. Wright JE: The treatment of childhood phimosis with topical steroid. Aust N Z J Surg. 1994; 64: 327-8. Erratum in: Aust N Z J Surg. 1995; 65: 698.

10. Jørgensen ET, Svensson A: The treatment of phimosis in boys, with a potent topical steroid (clobetasol propionate 0.05\%) cream. Acta Derm Venereol. 1993; 73: 55-6.

11. Van Howe RS: Cost-effective treatment of phimosis. Pediatrics. 1998; 102: E43.

12. Atilla MK, Dündaröz R, Odabaş 0 , Oztürk $H$, Akin R, Gökçay E: A nonsurgical approach to the treatment of phimosis: local nonsteroidal anti-inflammatory ointment application. J Urol. 1997; 158: 196-7.

13. Kikiros CS, Beasley SW, Woodward AA: The response of phimosis to local steroid application. Pediatr Surg Int. 1993; 8: 329-32. 
14. Yang SS, Tsai YC, Wu CC, Liu SP, Wang CC: Highly potent and moderately potent topical steroids are effective in treating phimosis: a prospective randomized study. J Urol. 2005; 173: 1361-3.

15. Lund L, Wai KH, Mui LM, Yeung CK: Effect of topical steroid on non-retractile prepubertal foreskin by a prospective, randomized, double-blind study. Scand J Urol Nephrol. 2000; 34: 267-9.

16. Marques TC, Sampaio FJ, Favorito LA: Treatment of phimosis with topical steroids and foreskin anatomy. Int Braz J Urol. 2005; 31: 370-4; discussion 374.

17. Mouton PR: Principles and practice of unbiased stereology. 1st edition, John Hopkins University Press. 2002.

18. Gundersen HJ, Bendtsen TF, Korbo L, Marcussen N, Møller A, Nielsen K, et al.: Some new, simple and efficient stereological methods and their use in pathological research and diagnosis. APMIS. 1988; 96: 379-94.
19. Sokal RR, Rohlf FJ: Statistical tables. In Freeman WH, eds., Biometry, 3rd ed. New York, 1995: 887-902.

20. Shankar KR, Rickwood AM: The incidence of phimosis in boys. BJU Int. 1999; 84: 101-2.

21. Lee KS, Koizumi T, Nakatsuji H, Kojima K, Yamamoto A, Kawanishi Y, et al.: Treatment of phimosis with betamethasone ointment in children. Nihon Hinyokika Gakkai Zasshi. 2001; 92: 619-23.

22. Marzaro M, Carmignola G, Zoppellaro F, Schiavon G, Ferro M, Fusaro F, et al.: Phimosis: when does it require surgical intervention?. Minerva Pediatr. 1997; 49: 245-8.

23. Cavalcanti AG, Costa WS, Baskin LS, McAninch JA, Sampaio FJ: A morphometric analysis of bulbar urethral strictures. BJU Int. 2007; 100: 397-402.

24. Cold CJ, Taylor JR: The prepuce. BJU Int. 1999; 83(Suppl 1): 34-44.
Correspondence address:

Dr. Luciano Alves Favorito Rua Professor Gabizo 104 / 201 Rio de Janeiro, RJ, 20271-320, Brazil

Fax: + $55213872-8802$

E-mail: lufavorito@yahoo.com.br 